\title{
ON THE THEOREM OF FRULLANI
}

\author{
JUAN ARIAS-DE-REYNA
}

(Communicated by R. Daniel Mauldin)

\begin{abstract}
We prove that, for every function $f: \mathbf{R}^{+} \rightarrow \mathbf{C}$ such that $(f(a x)-$ $f(b x)) / x$ is Denjoy-Perron integrable on $[0,+\infty)$ for every pair of positive real numbers $a, b$, there exists a constant $A$ (depending only on the values of $f(t)$ in the neighborhood of 0 and $+\infty)$ such that

$$
\int_{0}^{\infty} \frac{f(a x)-f(b x)}{x} d x=A \log \frac{a}{b} .
$$

To prove this assertion, we identify a Denjoy-Perron integrable function $f$ : $\mathbf{R} \rightarrow \mathbf{C}$ with a distribution. In this way, we obtain the main result of this paper: The value at 0 (in Lojasiewicz sense) of the Fourier transform of the distribution $f$ is the Denjoy-Perron integral of $f$. Assuming the Continuum Hypothesis, we construct an example of a non-Lebesgue measurable function that satisfies the hypotheses of the first theorem.
\end{abstract}

\section{INTRODUCTION}

The Italian mathematician G. Frullani, 1795-1834, reported to G. A. Plana, 1781-1864, the formula

$$
\int_{0}^{\infty} \frac{f(a x)-f(b x)}{x} d x=f(0) \log \frac{b}{a}
$$

in a letter dated in 1821 (cf. Edwards [7], vol. II, p. 339). Later, in 1828, Frullani published it [11], but apparently with an inadequate proof (cf. Tricomi [25], p. 49 and Ostrowski [20], p. 320). In 1823 and 1827, Cauchy gave a satisfactory proof of the formula

$$
\int_{0}^{\infty} \frac{f(a x)-f(b x)}{x} d x=[f(\infty)-f(0)] \log \frac{a}{b}
$$

under certain conditions on $f$ (cf. Ostrowski [20], p. 318-323). This same formula is attributed to E. B. Elliot [8] by Edwards [7] (vol. II, p. 339).

Cauchy's result has been fully generalized replacing the limits $f(0)$ and $f(\infty)$ by suitable mean values. It was K. S. K. Iyengar $[13,14]$ who first gave a formula of this type in 1940 . He proved that, being $f$ locally integrable on $(0,+\infty)$,

Received by the editors February 6, 1989 and, in revised form, July 12, 1989.

1980 Mathematics Subject Classification (1985 Revision). Primary 26A39, 26A42, 42A24, 46F12; Secondary $03 E 35$. 
the left side improper integral in (2) exists for every pair of real numbers $a, b$ if and only if the four limits

$$
\begin{array}{ll}
M^{*}(f)=\lim _{x \rightarrow+\infty} x \int_{x}^{\infty} \frac{f(t)}{t^{2}} d t, & \lim _{x \rightarrow+\infty} \int_{1}^{x} \frac{f(t)}{t^{2}} d t, \\
m^{*}(f)=\lim _{x \rightarrow 0^{+}} \frac{1}{x} \int_{0}^{x} f(t) d t, & \lim _{x \rightarrow 0^{+}} \int_{x}^{1} f(t) d t
\end{array}
$$

exist.

According to Ostrowski [21], his proof is not correct but his result was true. The first right proof is due to R. P. Agnew [1]. He proved that if

$$
L(\lambda)=\lim _{A \rightarrow \infty} \int_{A}^{A+\lambda} f(t) d t
$$

exists for each $\lambda$ in some set having positive measure, then $L(\lambda)$ exists for each $\lambda$ and $L(\lambda)=\lambda L$; moreover, the convergence is uniform over each finite interval. In 1949 Ostrowski [19] improved the theorem, putting it in what we consider its classical form:

Theorem. Assume $f(x)$ locally integrable in $(0,+\infty)$. Then we have, if both limits

$$
m(f)=\lim _{x \rightarrow 0^{+}} x \int_{x}^{1} \frac{f(t)}{t^{2}} d t, \quad M(f)=\lim _{x \rightarrow+\infty} \frac{1}{x} \int_{1}^{x} f(t) d t
$$

exist, then for any positive $a, b$,

$$
\int_{0}^{\infty} \frac{f(a x)-f(b x)}{x} d x=(M(f)-m(f)) \log \frac{a}{b} .
$$

Conversely, if the integral in (4) is convergent for a set of couples of positive values of $a$ and $b$, such that $a / b$ runs through a set of positive measure, both $M(f)$ and $m(f)$ exist.

A. Ostrowski said that the proof of this result was difficult and would be given elsewhere. The theorem was proved by R. P. Agnew in 1951 [2] and A. Ostrowski's proof appeared in 1976 [21]. Finally F. G. Tricomi ([25], pp. 4951 and [24]) and A. Ostrowski [19] generalized this formula in several ways.

Now, before introducing our theorem, let us consider the following: Let $\mathscr{E}$ be a linear space of real functions defined on $(0,+\infty)$ and $I: \mathscr{E} \rightarrow \mathbf{R}$ a linear form, that we denote by $I(f)=\int_{0}^{\infty} f(x) d x$, such that, if $f \in \mathscr{E}$ and $a>0$, then the function $g$ defined by $g(x)=f(a x)$ belongs to $\mathscr{E}$ and

$$
a \int_{0}^{\infty} f(a x) d x=\int_{0}^{\infty} f(x) d x
$$

Then, given $f:(0,+\infty) \rightarrow \mathbf{R}$ such that $(f(a x)-f(b x)) / x$ belongs to $\mathscr{E}$, for all positive $a$ and $b$, there exists a group homomorphism $\varphi: \mathbf{R}^{+} \rightarrow \mathbf{R}$ such that

$$
\int_{0}^{\infty} \frac{f(a x)-f(b x)}{x} d x=\varphi\left(\frac{a}{b}\right) .
$$

This is contained in essence in A. Ostrowski [21]. 
If we could prove that $\varphi$ is measurable, it would follow that $\varphi(t)=A \log t$, where $A$ is independent of $t$.

We thought about proving our theorem when we made the following remark about Frullani's integrals: In every concrete example, the axiom " $\varphi$ is measurable" is consistent with the ordinary theory of sets. This follows from Solovay's theorem [23]:

Theorem (Solovay). Assume that there exists an inaccessible cardinal. Then there is a model of ZFC in which every set of reals definable from a countable sequence of ordinals is Lebesgue measurable.

What we were searching for was a Frullani's "monster", that is, a function $f$ such that all the Frullani integrals, as in (5), exist but the homomorphism $\varphi$ is nonmeasurable. We managed to prove that such a monster does not exist, but doing so we obtained a theorem like Frullani's in which $f$ is not supposed locally integrable, even measurable, and therefore the value of the constant is not given by A. Ostrowski's formula (3).

To prove that our theorem is not contained in the classical one, we construct assuming the Continuum Hypothesis, a nonmeasurable function $f$ such that, for every $s, f(s+t)-f(t)=0$ a.e., so that we can apply our Theorem 2 to $f$. Changing the variable, we get an example for Theorem 2 where $f$ is not measurable. Martin's axiom suffices here because all we need show is that the union of less than the continuum sets of Lebesgue measure zero is of Lebesgue measure zero. As Martin's axiom has been treated at length (D. H. Fremlin [10]), we have detailed information concerning the hypotheses that are consistent with it. In particular, the conclusion of our Theorem 3 is also consistent with the negation of the Continuum Hypothesis.

In our exposition, we prove first a kind of theorem of Frullani for the Lebesgue integral. Note that this theorem cannot be applied to most examples in calculus texts, where we find improper but not Lebesgue integrals. That is the reason we later extend the theorem for the generalized Riemann integral [18], also known as the Denjoy-Perron integral.

The above example shows too that the known proofs of Frullani's theorem cannot be extended to our theorem. We give a new proof that we have not found in the bibliography (cf. J. Edwards [7], F. G. Tricomi [25], A. Ostrowski [20], G. M. Fichtenholz [9], G. Aumann and O. Haupt [5], H. Jeffreys and B. S. Jeffreys [15], T. M. Apostol [4]).

Solovay's theorem makes clear that we can get many generalized theorems changing the definition of the integral; for example, we would use the integral considered in Cheng-Ming Lee [16].

We want to add that the most important result of the paper is Theorem 6 (about the value at 0 of the Fourier transform of a Denjoy-Perron integrable function). 


\section{Frullani's theOREM FOR LEBESGUE INTEGRAL}

Theorem 1. Let $f:[0,+\infty) \rightarrow \mathbf{R}$ be a function such that $(f(a x)-f(b x)) / x$ is integrable on $[0,+\infty)$ for every pair of positive real numbers $a, b$. Then there exists a constant $A \in \mathbf{R}$ such that

$$
\int_{0}^{\infty} \frac{f(a x)-f(b x)}{x} d x=A \log \frac{a}{b}
$$

for every $a, b>0$.

Proof. First observe that the measurability of $f$ is not a hypothesis of the theorem. Let us take $x=e^{t}, \alpha=\log a$ and $\beta=\log b$. We obtain that (6) is equivalent to

$$
\int_{-\infty}^{+\infty}\left\{f\left(e^{t+\alpha}\right)-f\left(e^{t+\beta}\right)\right\} d t=A(\alpha-\beta) .
$$

So it suffices to prove the theorem that follows.

Theorem 2. Let $g: \mathbf{R} \rightarrow \mathbf{R}$ be a function such that $g(x+\alpha)-g(x+\beta)$ is Lebesgue integrable for every pair of real numbers $\alpha, \beta$. Then there exists a constant $A$ such that

$$
\int_{-\infty}^{+\infty}\{g(x+\alpha)-g(x+\beta)\} d x=A(\alpha-\beta) .
$$

Proof. Let $h_{\alpha}$ be defined by $h_{\alpha}(x)=g(x+\alpha)-g(x)$. We know that $h_{\alpha} \in L^{1}(\mathbf{R})$ for every real number $\alpha$.

Moreover, it is obvious that $h_{\alpha}(x)+h_{\beta}(x+\alpha)=h_{\alpha+\beta}(x)$.

Hence, $h_{\alpha}(x)+h_{\beta}(x+\alpha)=h_{\beta}(x)+h_{\alpha}(x+\beta)$.

All these functions belong to $L^{1}(\mathbf{R})$, so we can apply Fourier's transform and we ubtain

$$
\widehat{h_{\alpha}}(x)+e^{2 \pi i \alpha x} \widehat{h_{\beta}}(x)=\widehat{h_{\beta}}(x)+e^{2 \pi i \beta x} \widehat{h_{\alpha}}(x) .
$$

Thus we have

$$
\widehat{h_{\beta}}(x)=\frac{1-e^{2 \pi i \beta x}}{1-e^{2 \pi i \alpha x}} \widehat{h_{\alpha}}(x) .
$$

Let us observe that $\widehat{h_{\beta}}(x)$ is continuous and $G(\beta)=\int_{-\infty}^{+\infty}\{g(x+\beta)-g(x)\} d x=$ $\widehat{h_{\beta}}(0)$, and so

$$
G(\beta)=\lim _{x \rightarrow 0} \frac{1-e^{2 \pi i \beta x}}{1-e^{2 \pi i \alpha x}} \widehat{h_{c}}(x)=\frac{\beta}{\alpha} G(\alpha) .
$$

Now, if we choose $A=G(1)$, we have $G(\beta)=A \beta$, which leads to

$$
\begin{aligned}
(\alpha-\beta) A=G(\alpha-\beta) & =\int_{-\infty}^{+\infty}\{g(x+\alpha-\beta)-g(x)\} d x \\
& =\int_{-\infty}^{+\infty}\{g(x+\alpha)-g(x+\beta)\} d x,
\end{aligned}
$$

getting Theorem 2 . 
The next theorem, which contains the example we talked about in the introduction, must be compared with C. Burstin's theorem: A Lebesgue measurable function $f: \mathbf{R} \rightarrow \mathbf{R}$ having arbitrary small periods is a constant a.e. (cf. $\mathbf{R}$. Cignoli and J. Hounie [6] or J. M. Henle [12]).

Theorem 3. Assuming the Continuum Hypothesis, there exists a nonmeasurable function $f: \mathbf{R} \rightarrow \mathbf{R}$ such that, for every $s \in \mathbf{R}$,

$$
f(s+t)=f(t)
$$

for almost every $t \in \mathbf{R}$.

Note that, for every $s \in \mathbf{R}$, there is a different set of measure zero $Z_{s}$, so that (8) does not imply that $f$ is constant.

Proof. We shall construct $f$ as the characteristic function of some set $A \subset \mathbf{R}$. To construct $A$, we must first choose a certain Hamel basis of $\mathbf{R}$ over $\mathbf{Q}$. Let $\mathscr{K}$ be the set of all compact subsets of $\mathbf{R}$ of positive Lebesgue measure. The cardinality of $\mathscr{K}$ is the same as that of $\mathbf{R}$. So we can obtain a transfinite sequence $\left\langle K_{\alpha}: \alpha<\omega_{1}\right\rangle$ with the members of $\mathscr{K}$. Now, by transfinite induction, we define two transfinite sequences of real numbers $\left\langle x_{\alpha}: \alpha<\omega_{1}\right\rangle$ and $\left\langle y_{\alpha}: \alpha<\omega_{1}\right\rangle$ in the way that follows.

First, since $K_{0}$ is of positive measure, we can choose $z_{0}, z_{0}^{\prime}$ in $K_{0}$ linearly independent over the field $\mathbf{Q}$. We set $x_{0}=z_{0}, y_{0}=2 z_{0}^{\prime}$.

Now, suppose we have chosen the sequences $\left\langle x_{\beta}: \beta<\alpha\right\rangle$ and $\left\langle y_{\beta}: \beta<\alpha\right\rangle$ so that the set $B_{\alpha}=\left\{x_{\beta} \mid \beta<\alpha\right\} \cup\left\{y_{\beta} \mid \beta<\alpha\right\}$ is linearly independent over the field $\mathbf{Q}$. As we assumed the Continuum Hypothesis, the set $B_{\alpha}$ is countable and, since $K_{\alpha}$ has positive measure, we can select $z_{\alpha}$ and $z_{\alpha}^{\prime} \in K_{\alpha}$ such that $B_{\alpha} \cup\left\{z_{\alpha}, z_{\alpha}^{\prime}\right\}$ is linearly independent over the field $\mathbf{Q}$. Then we set $x_{\alpha}=z_{\alpha}$ and $y_{\alpha}=2 z_{\alpha}^{\prime}$.

Now we complete the linearly independent set $\left\{x_{\alpha} \mid \alpha<\omega_{1}\right\} \cup\left\{y_{\alpha} \mid \alpha<\omega_{1}\right\}$ to become an algebraic basis $B$ of $\mathbf{R}$ over $\mathbf{Q}$ and we form a transfinite sequence $\left\langle a_{\alpha} \mid \alpha<\omega_{1}\right\rangle$ with the members of $B$.

We can define now the set $A$. Observe that every real number $x \neq 0$ can be expressed in just one way as a finite linear combination

$$
x=\sum_{k=1}^{n} q_{\alpha_{k}} a_{\alpha_{k}}
$$

where $q_{\alpha_{k}} \in \mathbf{Q}$ does not vanish and $\alpha_{1}<\alpha_{2}<\cdots<\alpha_{n}$. Let $A$ be the set of those $x \in \mathbf{R}$ such that $q_{\alpha_{n}}$ is an integer.

Now we prove that, for every $s \in \mathbf{R}$, there exists $Z_{s}$ of measure zero such that $x \in \mathbf{R} \backslash Z_{s}$ implies that $x \in A$ if and only if $x+s \in A$. To see this, we note that, being

$$
s=\sum_{i=1}^{m} p_{i} a_{\beta_{i}}
$$


the representation of $s$ as a linear combination of elements of the basis $B$, every $x$ expressed as in (9) satisfies, whenever $\alpha_{n}>\beta_{m}$, that $x+s \in A$ if and only if $x \in A$ (we are supposing that $\beta_{m}>\beta_{i}$ if $i<m$ ). So that we can choose $Z_{s}$ as the linear span of the vectors of the set $\left\{a_{\xi} \mid \xi \leq \beta_{m}\right\}$. Since $Z_{s}$ is countable, it is a set of measure zero. This proves that the function $f=\chi_{A}$ verifies, for every $s \in \mathbf{R}$,

$$
f(s+t)=f(t) \text { a.e. }
$$

Let us see now that $f$ is non-Lebesgue measurable. We prove this assertion showing that, for every compact set $K$ of positive measure, we have

$$
K \cap A \neq \varnothing, \quad K \cap(\mathbf{R} \backslash A) \neq \varnothing .
$$

For, given such a compact $K$, there is an $\alpha<\omega_{1}$ such that $K=K_{\alpha}$. Now, the expressions

$$
z_{\alpha}=x_{\alpha}, \quad z_{\alpha}^{\prime}=\frac{1}{2} y_{\alpha}
$$

are the representations of the elements $z_{\alpha}$ and $z_{\alpha}^{\prime}$ of $K$ as linear combinations of elements of the basis $B$. So $z_{\alpha} \in A$ and $z_{\alpha}^{\prime} \notin A$. This proves (10).

\section{Distributions ANd the Denjoy-Perron integral}

It is usual to identify a locally integrable function $f: \mathbf{R} \rightarrow \mathbf{C}$ with the distribution $T_{f}: \mathscr{D} \rightarrow \mathbf{C}$ defined by $T_{f}(\varphi)=\int_{-\infty}^{+\infty} f(t) \varphi(t) d t$. Nevertheless, there is a problem here: there exist locally integrable functions $f: \mathbf{R} \rightarrow \mathbf{C}$ that admit a derivative $f^{\prime}(x)$ at all points $x \in \mathbf{R}$, without being $f^{\prime}$ locally integrable. In this situation we cannot identify $f^{\prime}$ with $D T_{f}$.

However, if we use the Denjoy-Perron integral instead of the Lebesgue integral, the problem vanishes. To prove this, we need a theorem of W. F. Pfeffer [22] on the Denjoy-Perron integral.

When we refer to the Denjoy-Perron integral we mean here the generalized Riemann integral as it is defined in R. M. McLeod [18]. This is justified, since both integrals are the same over compact intervals.

Theorem 4 (Pfeffer). If $f:[a, b] \rightarrow \mathbf{R}$ is Denjoy-Perron integrable on $[a, b]$ being

$$
F(x)=(D P) \int_{a}^{x} f(t) d t
$$

and $g$ is a function of bounded variation on $[a, b]$, then the product $f g$ is Denjoy-Perron integrable and

$$
(D P) \int_{a}^{b} f(t) g(t) d t=F(b) g(b)-F(a) g(a)-\int_{a}^{b} F(t) d g(t) .
$$

We denote by $(D P) \int_{a}^{b} f(t) d t$ the Denjoy-Perron integral of $f$ on $[a, b]$.

We say that $f: \mathbf{R} \rightarrow \mathbf{C}$ is a locally Denjoy-Perron integrable function when, for every bounded interval $[a, b] \subset \mathbf{R}, f$ is Denjoy-Perron integrable on 
$[a, b]$. If $f: \mathbf{R} \rightarrow \mathbf{C}$ is locally Denjoy-Perron integrable, we define $T_{f}: \mathscr{D} \rightarrow \mathbf{C}$ by

$$
T_{f}(\varphi)=(D P) \int_{-\infty}^{+\infty} f(t) \varphi(t) d t .
$$

Note that $T_{f}$ is a distribution because if $\sup (\varphi) \subset(a, b)$, by Theorem 4, we can write

$$
T_{f}(\varphi)=(D P) \int_{-\infty}^{+\infty} f(t) \varphi(t) d t=(D P) \int_{a}^{b} f(t) \varphi(t) d t=-\int_{a}^{b} F(t) d \varphi(t)
$$

and, since $F$ is continuous (see R. M. McLeod [18] p. 58), we have

$$
\left|T_{f}(\varphi)\right|=\left|-\int_{a}^{b} F(t) \varphi^{\prime}(t) d t\right| \leq M_{a, b} \sup \left|\varphi^{\prime}(t)\right| .
$$

Now, if $f$ is differentiable at every point, $f^{\prime}$ is locally Denjoy-Perron integrable (see R. M. McLeod [18], p. 27). Moreover, $T_{f^{\prime}}=D T_{f}$, because if $\varphi \in \mathscr{D}$, we have by Theorem 4:

$$
\left\langle T_{f^{\prime}},(\varphi)\right\rangle=(D P) \int_{-\infty}^{+\infty} f^{\prime}(t) \varphi(t) d t=-\int_{-\infty}^{+\infty} f(t) \varphi^{\prime}(t) d t=\left\langle D T_{f}, \varphi\right\rangle .
$$

Theorem 5. Let $f: \mathbf{R} \rightarrow \mathbf{C}$ Denjoy-Perron integrable. Then $f$ is a tempered distribution.

Proof. Put $F(x)=(D P) \int_{0}^{x} f(t) d t$ for every $x \in \mathbf{R}$.

By Theorem 4 , for every $\varphi \in S$,

$$
(D P) \int_{a}^{b} f(t) \varphi(t) d t=F(b) \varphi(b)-F(a) \varphi(a)-\int_{a}^{b} F(t) d \varphi(t) .
$$

Observe that, as $F$ is bounded and continuous and $\varphi \in S$, the RiemannStieltjes integral $\int_{-\infty}^{+\infty} F(t) d \varphi(t)$ is defined, and

$$
\lim _{b \rightarrow+\infty} F(b) \varphi(b)=\lim _{a \rightarrow-\infty} F(a) \varphi(a)=0 .
$$

So $f(t) \varphi(t)$ is Denjoy-Perron integrable on $\mathbf{R}$ and

$$
(D P) \int_{-\infty}^{+\infty} f(t) \varphi(t) d t=-\int_{-\infty}^{+\infty} F(t) \varphi^{\prime}(t) d t .
$$

Now, since $\left|\int_{-\infty}^{+\infty} F(t) \varphi^{\prime}(t) d t\right| \leq M \sup _{t \in \mathbf{R}}\left|\varphi^{\prime}(t)\right|$, we see that the linear form $\varphi \mapsto(D P) \int_{-\infty}^{+\infty} f(t) \varphi(t) d t$ is a tempered distribution.

As we want to extend the proof of Theorem 2 to the Denjoy-Perron integral instead of the Lebesgue integral, we need S. Lojasiewicz's definition [17] of the value of a distribution at a point. It is said that a distribution $T$ has a value at a point $a$ when there exists the limit

$$
\lim _{\lambda \rightarrow 0} T(a+\lambda x)
$$

in the space $\mathscr{D}^{\prime}$ of distributions. This limit, when it exists, is a constant distribution, and we say that this constant is the value of $T$ at $a$ (see $\mathrm{S}$. Lojasiewicz [17] or P. Antosik, J. Mikusiński and R. Sikorski [3]). 
Theorem 6. Let $f: \mathbf{R} \rightarrow \mathbf{C}$ be Denjoy-Perron integrable. Then the Fourier transform of $f$ has the value $(D P) \int_{-\infty}^{+\infty} f(t) d t$ at 0 .

Proof. Fix $\varphi \in \mathscr{D}$. We must prove

$$
\lim _{\lambda \rightarrow 0}\langle\hat{f}(\lambda x), \varphi(x)\rangle=\left((D P) \int_{-\infty}^{+\infty} f(t) d t\right)\left(\int_{-\infty}^{+\infty} \varphi(t) d t\right) .
$$

Let us observe that

$$
\langle\hat{f}(\lambda x), \varphi(x)\rangle=\left\langle\hat{f}(x), \varphi\left(\frac{x}{\lambda}\right) \frac{1}{\lambda}\right\rangle=\left\langle f(t), \frac{1}{\lambda} \hat{\varphi}\left(\frac{x}{\lambda}\right)(t)\right\rangle,
$$

and since

$$
\int_{-\infty}^{+\infty} \frac{1}{\lambda} \varphi\left(\frac{1}{\lambda}\right) e^{-2 \pi i x t} d x=\int_{-\infty}^{+\infty} \varphi(x) e^{-2 \pi i x \lambda t} d t=\hat{\varphi}(\lambda t)
$$

we have

$$
\langle\hat{f}(\lambda x), \varphi(x)\rangle=\langle f(t), \hat{\varphi}(\lambda t)\rangle=(D P) \int_{-\infty}^{+\infty} f(t) \hat{\varphi}(\lambda t) d t .
$$

So it remains to prove that

$$
\lim _{\lambda \rightarrow 0}(D P) \int_{-\infty}^{+\infty} f(t) \hat{\varphi}(\lambda t) d t=\hat{\varphi}(0)\left((D P) \int_{-\infty}^{+\infty} f(t) d t\right)
$$

where $\hat{\varphi} \in S$.

To prove (11), we can replace $f$ by $f+g$ where $g$ is any Lebesgue integrable function. We can therefore assume that

$$
(D P) \int_{0}^{+\infty} f(t) d t=(D P) \int_{-\infty}^{0} f(t) d t=0 .
$$

If we put $F(x)=(D P) \int_{0}^{x} f(t) d t$, then $\lim _{x \rightarrow+\infty} F(x)=\lim _{x \rightarrow-\infty} F(x)=0$. We know that $\hat{\varphi}$ is of bounded variation. So, given $\varepsilon>0$, there exists $M>0$ such that, for every $y>M$ and $\lambda \in \mathbf{R}$, we have

$$
\begin{gathered}
\left|\int_{y}^{+\infty} F(t) d \hat{\varphi}(\lambda t)\right|<\varepsilon, \quad\left|\int_{-\infty}^{-y} F(t) d \hat{\varphi}(\lambda t)\right|<\varepsilon, \\
|F(y) \hat{\varphi}(\lambda y)|<\varepsilon, \quad \text { and } \quad|F(-y) \hat{\varphi}(-\lambda y)|<\varepsilon .
\end{gathered}
$$

Moreover, by Henstock's lemma (McLeod [18], p. 74), there exists a function $\delta: \overline{\mathbf{R}} \rightarrow \mathscr{I}$, being $\mathscr{I}$ the set of closed intervals of $\overline{\mathbf{R}}$, verifying that $\xi \in \delta(\xi)$ and the condition that follows:

If $a=t_{0}<t_{1}<\cdots<t_{n}=b, \xi_{k} \in\left[t_{k-1}, t_{k}\right] \subset \delta\left(\xi_{k}\right),(b,+\infty) \subset \delta(+\infty)$ and $(-\infty, a) \subset \delta(-\infty)$, then

$$
\sum_{k=1}^{n}\left|f\left(\xi_{k}\right)\left(t_{k}-t_{k-1}\right)-(D P) \int_{t_{k-1}}^{t_{k}} f(t) d t\right| \leq \varepsilon
$$

We can also assume that, for every $\xi \in \mathbf{R}$, the oscillation of $F$ on $\delta(\xi)$ is less than $\varepsilon /(1+V)$, where $V=\int_{-\infty}^{+\infty}\left|\varphi^{\prime}(t)\right| d t$ is the total variation of $\varphi$ over $\mathbf{R}$, and such that $\delta(+\infty) \subset[M,+\infty]$ and $\delta(-\infty) \subset[-\infty,-M]$. 
Then, if $a=t_{0}<t_{1}<\cdots<t_{n}=b, \xi_{k} \in\left[t_{k-1}, t_{k}\right] \subset \delta\left(\xi_{k}\right),(b,+\infty) \subset$ $\delta(+\infty)$ and $(-\infty, a) \subset \delta(-\infty)$, we have

$$
\begin{aligned}
&\left|\sum_{k=1}^{n} f\left(\xi_{k}\right) \hat{\varphi}\left(\lambda \xi_{k}\right)\left(t_{k}-t_{k-1}\right)-\int_{-\infty}^{+\infty} F(t) d \hat{\varphi}(\lambda t)\right| \\
& \leq 4 \varepsilon+\mid \sum_{k=1}^{n} f\left(\xi_{k}\right) \hat{\varphi}\left(\lambda \xi_{k}\right)\left(t_{k}-t_{k-1}\right) \\
&-\left\{F(b) \hat{\varphi}(\lambda b)-F(a) \hat{\varphi}(\lambda a)+\int_{a}^{b} F(t) d \hat{\varphi}(\lambda t)\right\} \mid \\
& \leq 4 \varepsilon+\mid \sum_{k=1}^{n} f\left(\xi_{k}\right) \hat{\varphi}\left(\lambda \xi_{k}\right)\left(t_{k}-t_{k-1}\right)-\left\{\sum_{k=1}^{n} F\left(t_{k}\right) \hat{\varphi}\left(\lambda t_{k}\right)-F\left(t_{k-1}\right) \hat{\varphi}\left(\lambda t_{k-1}\right)+\int_{a}^{b} F(t) d \hat{\varphi}(\lambda t)\right\} \mid \\
& \leq 4 \varepsilon+\mid \sum_{k=1}^{n}\left\{f\left(\xi_{k}\right)\left(t_{k}-t_{k-1}\right)-\left(F\left(t_{k}\right)-F\left(t_{k-1}\right)\right)\right\} \hat{\varphi}\left(\lambda \xi_{k}\right) \\
& \quad-\sum_{k=1}^{n} F\left(t_{k}\right)\left(\hat{\varphi}\left(\lambda t_{k}\right)-\hat{\varphi}\left(\lambda \xi_{k}\right)\right)-\sum_{k=1}^{n} F\left(t_{k-1}\right)\left(\hat{\varphi}\left(\lambda \xi_{k}\right)-\hat{\varphi}\left(\lambda t_{k-1}\right)\right) \\
& \leq 4 \varepsilon+\varepsilon \sup |\hat{\varphi}(t)|+\frac{\varepsilon}{1+V} V \leq 5 \varepsilon+\varepsilon \sup _{t \in \mathbb{R}}^{b}|\hat{\varphi}(t)| . \quad F(t) d \hat{\varphi}(\lambda t) \mid
\end{aligned}
$$

It is clear that, for all $\lambda$ sufficiently small,

$$
\left|\hat{\varphi}(0) \sum_{k=1}^{n} f\left(\xi_{k}\right)\left(t_{k}-t_{k-1}\right)-\int_{-\infty}^{+\infty} F(t) d \hat{\varphi}(\lambda t)\right|<6 \varepsilon+\varepsilon \sup _{t \in \mathbf{R}}|\hat{\varphi}(t)| .
$$

Now, if we choose $\delta$ such that

$$
\left|\sum_{k=1}^{n} f\left(\xi_{k}\right)\left(t_{k}-t_{k-1}\right)-(D P) \int_{-\infty}^{+\infty} f(t) d t\right|<\frac{\varepsilon}{\sup _{t \in \mathbf{R}}|\hat{\varphi}(t)|},
$$

we will have

$$
\left|\hat{\varphi}(0)(D P) \int_{-\infty}^{+\infty} f(t) d t-\int_{-\infty}^{+\infty} F(t) d \hat{\varphi}(\lambda t)\right|<7 \varepsilon+\varepsilon \sup _{t \in \mathbf{R}}|\hat{\varphi}(t)|
$$

and the proof is completed.

\section{Frullani's theorem for the Denjoy-Perron integral}

Theorem 7. Let $f:[0,+\infty) \rightarrow \mathbf{R}$ be a function such that $(f(a x)-f(b x)) / x$ is Denjoy-Perron integrable over $[0,+\infty)$ for every pair of positive real numbers 
$a, b$. Then there exists a constant $A$ satisfying

$$
(D P) \int_{0}^{+\infty} \frac{f(a x)-f(b x)}{x} d x=A \log \left\{\frac{a}{b}\right\} .
$$

Proof. As in the proof of Theorem 1, changing variables (McLeod [18], p. 64), it suffices to prove the following theorem.

Theorem 8. Let $g: \mathbf{R} \rightarrow \mathbf{R}$ be a function such that $g(x+\alpha)-g(x+\beta)$ is Denjoy-Perron integrable over $\mathbf{R}$ for every pair of real numbers $\alpha, \beta$. Then there exists a constant $A$ satisfying

$$
(D P) \int_{-\infty}^{+\infty}\{g(x+\alpha)-g(x+\beta)\} d x=A(\alpha-\beta) .
$$

Proof. The proof of this theorem is analogous with that of Theorem 2. Put $h_{\alpha}(x)=g(x+\alpha)-g(x)$. Then

$$
h_{\alpha}(x)+h_{\beta}(x+\alpha)=h_{\beta}(x)+h_{\alpha}(x+\beta) .
$$

Applying the Fourier transform we obtain

$$
\widehat{h_{\alpha}}(x)+e^{2 \pi i \alpha x} \widehat{h_{\beta}}(x)=\widehat{h_{\beta}}(x) \hat{h}_{\beta}(x)+e^{2 \pi i \beta x} \hat{h}_{\alpha}(x)^{2 \pi i \beta x} \widehat{h_{\alpha}}(x) .
$$

Thus, in a neighborhood of 0 , we have

$$
\widehat{h_{\beta}}(x)=\frac{1-e^{2 \pi i \beta x}}{1-e^{2 \pi i \alpha x}} \widehat{h_{\alpha}}(x) .
$$

Since the function $\left(1-e^{2 \pi i \beta x}\right) /\left(1-e^{2 \pi i \kappa x}\right)$ is infinitely differentiable, we can apply the theorem of Lojasiewicz [17], p. 14 (see also P. Antosik, J. Mikusiński, and R. Sikorski [3], p. 43). So

$$
\hat{h}_{\beta}(0)=\frac{\beta}{\alpha} \hat{h}_{\alpha}(0) .
$$

We now apply Theorem 6 and obtain

$$
(D P) \int_{-\infty}^{+\infty}\{g(x+\beta)-g(x)\} d x=\frac{\beta}{\alpha}(D P) \int_{-\infty}^{+\infty}\{g(x+\alpha)-g(x)\} d x .
$$

We conclude the proof as in Theorem 3 .

Theorem 9. Let $f_{1}, f_{2}: \mathbf{R} \rightarrow \mathbf{R}$ be functions such that $f_{1}(x+\alpha)-f_{1}(x+\beta)$ and $f_{2}(x+\alpha)-f_{2}(x+\beta)$ are Denjoy-Perron integrable for every pair of real numbers $\alpha, \beta$. Assume that there exists a constant $M>0$ such that $f_{1}(x)=f_{2}(x)$ for every $x$ such that $|x|>M$. Then

$$
(D P) \int_{-\infty}^{+\infty}\left\{f_{1}(x+\alpha)-f_{1}(x+\beta)\right\} d x=(D P) \int_{-\infty}^{+\infty}\left\{f_{2}(x+\alpha)-f_{2}(x+\beta)\right\} d x,
$$

for every real number $\alpha$. 
Proof. Let $f(x)=f_{1}(x)-f_{2}(x)$. Then $f$ satisfies the conditions of Theorem 8 . Besides, $f$ is Denjoy-Perron integrable. In fact, $f(x)-f(x+2 M)=f(x)$ for every $|x|<M$. Now, it is clear that

$$
(D P) \int_{-\infty}^{+\infty}\{f(x+\alpha)-f(x+\beta)\} d x=0
$$

and this proves the theorem.

\section{REFERENCES}

1. R. P. Agnew, Limits of integrals, Duke Math. J. 9 (1942), 10-19.

2. __, Mean values and Frullani integrals, Proc. Amer. Math. Soc. 2 (1951), 237-241.

3. P. Antosik, J. Mikusiński, and R. Sikorski, Theory of distributions. The sequential approach, Elsevier, Amsterdam, 1973.

4. T. M. Apostol, Mathematical analysis, 2nd ed., Addison Wesley, Reading, MA, 1974.

5. G. Aumann and O. Haupt, Einführung in die reelle Analysis, vol. I, Walter de Gruyter, Berlin, 1974.

6. R. Cignoli and J. Hounie, Functions with arbitrarily small periods, Amer. Math. Monthly 85 (1978), 582-584.

7. J. Edwards, A treatise on the integral calculus, Chelsea, New York, (1st ed., 1922).

8. E. B. Elliot, Educational Times, 1875.

9. G. M. Fichtenholz, Differential- und Integralrechnung, vol. II, V.E.B. Deutscher Verlag, Berlin, 1974.

10. D. H. Fremlin, Consequences of Martin's Axiom, Cambridge University Press, Cambridge, 1984.

11. G. Frullani, Sopra gli integrali definiti, Mem. Societa Ital. d. Scienze 20 (1828), 44-48.

12. J. M. Henle, Functions with arbitrarily small periods, Amer. Math. Monthly 87 (1980), 816.

13. K. S. K. Iyengar, On Frullani integrals, J. Indian Math. Soc. (2), 4 (1940), 145-150.

14. __, On Frullani integrals, Proc. Cambridge Phil. Soc. 37 (1941), 9-13.

15. H. Jeffreys and B. S. Jeffreys, Methods of mathematical physics, 3rd ed., Cambridge University Press, 1956.

16. C. M. Lee, Generalizations of Cesàro continuous functions and integrals of Perron type, Trans. Amer. Math. Soc. 266 (1981), 461-481.

17. S. Lojasiewicz, Sur la valeur et la limite d'une distribution en un point, Studia Math. 16 (1957), 1-36.

18. R. M. McLeod, The generalized Riemann integral, Math. Assoc. of America (1980).

19. A. Ostrowski, On some generalizations of the Cauchy-Frullani integral, Proc. Nat. Acad. Sci. U.S.A. 35 (1949), 612-616. Collected Mathematical Papers, IV, pp. 148-152.

20. __ Vorlesungen über Differential- und Integralrechnung, vol. III, Verlag Birkhäuser, Basel, (1954).

21. __, On Cauchy-Frullani integrals, Comment. Math. Helv. 51 (1976), 57-91. Collected Mathematical Papers, IV, pp. 349-383.

22. W. F. Pfeffer, Integration by parts for the generalized Riemann-Stieltjes integral, J. Austral. Math. Soc. Ser. A34 (1983), 229-233.

23. R. M. Solovay, A model of set theory in which every set of reals is Lebesgue measurable, Ann. of Math. 92 (1970), 1-56.

24. F. G. Tricomi, On the theorem of Frullani, Amer. Math. Monthly 58 (1951).

25. ___ Esercizi e complementi di Analisi Matematica, Parte Seconda, Terza Edizioni, C.E.D.A.M., Padova, 1960.

Universidad de Sevilla, Facultad de Matematicas, Apdo. 1160, 41080 Sevilla, Spain 